\title{
Correction to: A Core-Envelope Massive Distribution with a Parabolic Density Distribution in the Core
}

\author{
P. S. Negi ${ }^{1}$ - A. K. Pande ${ }^{1} \cdot$ M. C. Durgapal ${ }^{1}$ \\ Published online: 4 October 2019 \\ (c) Springer Science+Business Media, LLC, part of Springer Nature 2019
}

\section{Correction to: General Relativity and Gravitation, Vol. 22, No. 7, 1990 https://doi.org/10.1007/BF00764153}

The study carried out by Negi et al. [1] deals with the construction of a core-envelope model of static and spherical mass distribution characterized by exact solutions of Einstein's field equations. The core of the model is described by Tolman's VII solution [2] matched smoothly at the core-envelope boundary. The region of the envelope is described by Tolman's V solution [2] which is finally matched to the vacuum Schwarzschild solution. The core-envelope boundary of the model is assured by matching of all the four variables-pressure $(P)$, energy density $(E)$ and both of the metric parameters $v$ and $\lambda$ with recourse to the computational method. However, it appears that while computing the core-envelope boundary and other parameters by using Eqs. (19)-(22) and thereafter following the expression for $w_{b}$, some error occurred in the computation of Negi et al. [1] which has affected the results of this paper significantly. I have found that this matching can be ensured for the values of $n$ in the range $0<n \leq(3 / 4)$ and that the matching does not exist for the values of $n>(3 / 4)$. The last claim is not in agreement with the claim made in the study of Negi et al. [1] that the matching also exists for the value of $n=1[u=(1 / 3)]$. I have carried out this matching for the values of $n=(1 / 2)$ and $n=(3 / 4)$ [that is for $u$ values $=0.25$ and 0.30 , respectively], and the results of this study will be presented in a separate paper. The matching for other allowed values of $n$ in the range prescribed above can also be done likewise.

Deceased: A. K. Pande and M. C. Durgapal.

The original article can be found online at https://doi.org/10.1007/BF00764153.

$\triangle$ P. S. Negi

psneginainital63@gmail.com

1 Department of Physics, Kumaun University, Nainital, India 


\section{References}

1. Negi, P.S., Pande, A.K., Durgapal, M.C.: Gen. Relativ. Gravit. 22, 735 (1990)

2. Tolman, R.C.: Phys. Rev. 55, 364 (1939)

Publisher's Note Springer Nature remains neutral with regard to jurisdictional claims in published maps and institutional affiliations. 